\title{
Overview of Key Technologies of Data Transmission in Complex Cognitive Wireless Networks
}

\author{
Xiao Shoubai \\ (NanChang Institute of Science and Technology, Nanchang 330108)
}

Keywords: Cognitive network; Data transmission; Overview

\begin{abstract}
This paper takes the application of complex network theory approach in cognitive wireless networks as the basic background, and discusses the key technologies of cognitive wireless networks based on complex network theory. The basic goal is to optimize the cognitive wireless network's end-to-end performance. . This paper aims at the theoretical and applied research needs of cognitive wireless networks. The results of previous pre-researches are the basic starting point to study the relationship between network transport performance optimization, topology control and routing strategies based on complex network theory in cognitive wireless networks. The basic breakthroughs provide new research methods, research ideas and theories for the study of topology control theory and routing strategies in cognitive wireless networks.

With the rapid development of wireless communication technology, a new generation of wireless networks is gradually moving toward diversification, isomerization, and intelligence. An important development trend of the future network is broadband and diversification of services. The main reasons for limiting it are the shortage of spectrum resources and the lack of flexibility of the existing fixed spectrum management modes. The dynamically changing heterogeneous network environment further exacerbates the contradiction between resource shortages and business requirements, thus seriously restricting the deployment and operation of existing and future wireless networks. However, actual measurement data shows that the wireless spectrum is underutilized most of the time and in some areas. In order to increase the utilization of the existing spectrum, Dr. Mitola proposed cognitive radio technology in 1999. Cognitive radios use "secondary use" of licensed spectrum. Dynamic spectrum access allows non-authorized users to perceive and access current idle frequency bands. Cognitive radio networks (CRNs) adopting cognitive radio technology not only include cognitive radio technology, but also put forward more and higher requirements at the network level. The essence is to incorporate cognitive characteristics into the overall wireless communication network.
\end{abstract}

\section{Research Significance}

At present, there are many researchers researching cognitive wireless networks, CORVUS in Berkeley, University of California, KNOWS system developed by the University of Maryland and Microsoft Research, OCRA project proposed by George Tech, and DRiVE project in EU. With the development of project research, the technology has made some progress in spectrum sensing, network framework, and protocol design. The IEEE has also established an IEEE 802.22 working group for the standardization of this technology. China also attaches great importance to cognitive wireless technology. Both the 863 plan in 2005 and the 973 plan in 2009 support the research of wireless cognitive technology.

Compared with traditional wireless networks, cognitive wireless networks also have some new problems that need further exploration and in-depth research. For example, the cognitive wireless network architecture adaptability and the multi-domain environment based on this, the cognitive, network resource distribution management and other issues. In recent years, in response to these problems, domestic and foreign researchers and research institutes have carried out related research, such as spectrum detection/awareness, spectrum sharing, spectrum management, topology control, routing protocols, and transport layer protocols.

Complex networks are ubiquitous in a large number of real networks. With the rapid development of network technologies, the network structure is becoming increasingly complex, 
making it difficult for traditional stochastic network models to describe their topological characteristics objectively. Therefore, the theory of complex networks provides a new perspective and thinking for the study of wireless networks. The relationship between wireless networks and complex networks and the application of complex network theory in wireless networks have attracted the attention of some scholars.

\section{Research Status}

Currently, as the demand for wireless communication services grows rapidly, available spectrum resources become increasingly scarce. People use the advanced wireless communication theories and technologies, such as link adaptation technology and multi-antenna technology, to increase the efficiency of spectrum utilization to ease the pressure of spectrum shortage. At the same time, the actual measurement results show that there is a very serious phenomenon of idle and wasted spectrum resources allocated. The statistical results show that the global licensed frequency band, especially the low frequency band with better signal propagation characteristics, has extremely low spectrum utilization. In the fixed spectrum allocation strategy, authorized users have exclusive rights to the spectrum. Even if the spectrum is idle, other users cannot use it. In fact, the authorized user's service is bursty, its use of the spectrum is intermittent, and the spectrum is often idle. Therefore, the efficient and dynamic allocation and management of spectrum resources is an important way to significantly increase the utilization of spectrum resources, which is conducive to the resolution of the "shortage" and "waste" of the spectrum.

Because the cognitive radio technology can utilize the cognitive function of the node, perceive the wireless environment, and achieve the dynamic spectrum access through intelligent analysis and decision, it has received great attention from the industry. In 1999, Mitola put forward the concept of cognitive radio based on Software Defined Radio (SDR). He believes that wireless terminals should have sufficient intelligence or cognitive ability to obtain optimal strategies by detecting, analyzing, learning, reasoning, and planning by integrating the history and current conditions of the surrounding wireless environment. After obtaining the environmental information, the system reconfigures the wireless link transmission parameters, such as frequency, modulation method, transmission power, etc., to realize high-quality wireless transmission.

\section{Cognitive Wireless Network Topology Control}

Topology is a fundamental problem in communication networks. The so-called topology control, in short, is to provide a good network topology for the network. Topological control research is not only to make it can save energy effectively, thus prolonging the network's lifetime, but also has important significance for reducing communication interference, increasing network capacity and improving the efficiency of MAC protocol and routing protocol. In a multi-hop wireless network, data packets reach a destination node via multi-hop continuous wireless links. The topology of a multi-hop wireless network refers to a set of communication link combinations between node pairs obtained through an explicit/implicit routing mechanism. . An important difference between cognitive wireless networks and cognitive radios is that cognitive wireless networks aim at end-to-end performance. At this point, topology control is particularly important in the network it constitutes.

In a cognitive wireless network, in order to avoid PU interference, the SU needs to give up the corresponding channel when the PU accesses the channel. This has a certain impact on the overall performance of the cognitive wireless network. The reasons are: (1) The active area of the PU is generally larger than the active area of the SU, so that the presence of the PU affects the SU that will transmit data; (2) In order to obtain other available spectrum, the SU needs to spend some time to sense the free spectrum, Neighbor discovery and channel switching; (3) Channel switching will bring some chain reaction, such as cascade switching of multiple SUs. Because the activity of the PU is more difficult to predict, in particular, in a multi-hop cognitive wireless network, if the connection between all the links is affected by the activity of the PU, the network partition is highly 
likely to occur, resulting in packet loss and The end-to-end delay of the affected SU data packets reduces the overall performance of the cognitive wireless network. Therefore, in a cognitive wireless network, the topology control is more complicated, and it is affected by the interference of the PU and the available links in a period of time.

\section{Cognitive Radio Network Routing Protocol}

Routing technology is the key technology at the network layer. For cognitive radio systems, the spectrum resources used by users are uncertain. Due to the time and spatial discontinuity of the spectrum, the nodes that are communicating may break the connection due to the appearance of the PU, and the available frequency set of each node. It is dynamic and heterogeneous, making the routing in cognitive radio networks much more complex than traditional networks. In addition to considering the energy, distance, etc., the route design of the cognitive network must also consider the perceptual characteristics of the SU, the interference to the PU and other factors, so that the path of the SU transmission data can avoid the PU. In addition, there may be multiple available frequency resources for the SU. How to effectively coordinate the frequency resources between source destination nodes is also a factor that should be considered. Therefore, the routing protocol of the traditional multi-hop wireless network cannot be directly applied to the CRN, and a new routing protocol needs to be designed for it.

\section{Research on Wireless Network Based on Complex Network Theory}

In recent years, as a new research field, complex networks can be used to describe a wide variety of real systems in nature, attracting the attention of more and more researchers at home and abroad. Complex networks have small-world characteristics and scale-free characteristics, and their basic theories are penetrating social networks, computer networks, power networks, and protein networks. With the development of information technology, the network becomes more and more complex. How to seek a research method that can meet the needs of users and save network resources to improve network performance in a complicated and changeable network environment has become a topic of concern to researchers. . As a tool to study complex systems, complex networks can be used to model and analyze complex information networks, draw useful conclusions, guide people's practices, and improve the overall performance of information networks.

With the development of wireless communication technologies, wireless networks have become more and more complicated. It manifests itself in the complexity of the network structure, the dynamic changes of the network topology, the complexity of the nodes, and the rapid development of wireless access technologies, which makes it possible that there may be multiple heterogeneous nodes in a network, and the state of the nodes changes with time. The application of complex networks in wireless networks has attracted the attention of some scholars.

\section{Core Problems and Research Ideas in the Current Status of Research}

To sum up, problems such as topology control problems, routing problems, and performance research of cognitive wireless networks based on complex network theory are undoubtedly the focus of attention in cognitive wireless networks at home and abroad. However, most of the research work lacks systemic research. The ideas and research methods do not fully take into account the particularities of cognitive wireless networks, such as spectrum opportunistic access, PU activity, link indirect connectivity, and diversification of SU requirements. This is exactly what the project is trying to do in-depth research.

Through observation and research, it is found that social networks can be found more successfully through the characteristics of complex networks. Through the discovery of social networks, we can conduct detailed user behavior analysis and research, so as to statistically evaluate and evaluate the application traffic and its traffic characteristics. Knowledge of user behaviors, etc., discovers and constructs network topologies that are commensurate with them, which will affect 
network topology control and structural stability, thus helping to determine the management link relationships, spectrum resources, etc. This research will be studied in depth. Cognitive wireless networks provide new theoretical foundations and research methods. Although the previous study of this project team has done some work in this area, it is only an exploratory study and lacks precision and depth. There are still many problems to be solved.

\section{Acknowledgement}

Science and Technology research project of Jiangxi Provincial Education Department (No.GJJ171095), and Nanchang Key Laboratory of Intelligent Building Network Engineering.

\section{References}

[1] A. Helmy. Small worlds in Wireless Networks. IEEE Communications Letters, 2003, 7(10): 490-492.

[2] FCC, Notice of Proposed Rule Making and Order. ET Docket No.03-322, Dec.2003.

[3] G. Sharma, R. Mazumdar. Hybrid sensor networks: a small world. ACM MobiHoc, 2005:366-377.

[4] J. Mitola. The software radio architecture. IEEE Communications Magazine, 1995, 33(5):26-38.

[5] I. F. Akyildiz, W. Y. Lee, M. C. Vuran et al. Next generation/dynamic spectrum access/cognitive radio wireless networks: A survey. Computer Networks, 2007, 50(13): 2127-2159.

[6] A. Rajaee, M. Saedy, A. Sahebalam. Competitive spectrum sharing for cognitive radio on Scale-Free wireless networks. IEEE, IWCMC, 2011:455-459.

[7] J. Tang, G. Xue, W. Zhang, Interference-Aware Topology Control and QoS Routing in Multi-Channel Wireless Mesh Networks, ACM MobiHoc, 2005:68-77.

[8] D. J. Watts, S. H. Strogatz. Collective dynamics of 'small-world' networks. Nature, 1998, 393, (6684): 440-442.

[9] J. Mitola, G.Q. Maguire. Cognitive radio: Making software radios more personal. IEEE Personal Communications, 1999, 6(4):13-18.

[10] P. Y. Chen, K.C. Chen. Information Epidemics in Complex Networks with Opportunistic Links and Dynamic Topology. IEEE GLOBECOM, 2010:1-6. 